\section{Incapacidades físicas em pessoas acometidas pela hanseníase no período pós-alta da poliquimioterapia em um município no Norte do Brasil}

\author{
Physical disabilities in leprosy patients after \\ discharge from multidrug therapy in \\ Northern Brazil
}

\author{
Incapacidades físicas en personas afectadas por \\ la hanseniasis en el período post alta de la \\ poliquimioterapia en un municipio en el \\ norte de Brasil
}

Lorena Dias Monteiro 1,2 Carlos Henrique Morais de Alencar 1,3 Jaqueline Caracas Barbosa 1 Katiane Pereira Braga 4 Milene Damous de Castro 3 Jorg Heukelbach 1,5

\footnotetext{
1 Universidade Federal do Ceará, Fortaleza, Brasil. 2 Secretaria de Estado da Saúde do Tocantins, Araguaína, Brasil. ${ }_{3}^{3}$ Swiss Tropical and Public Health Institute, University of Basel, Basel, Switzerland. ${ }^{4}$ Instituto Federal de Educação, Ciência e Tecnologia do Tocantins, Palmas, Brasil.

5 School of Public Health, Tropical Medicine and Rehabilitation Sciences, James Cook University, Townsville, Australia.

Correspondência C. H. M. Alencar Universidade Federal do Ceará.

Rua Professor Costa Mendes 1608, Fortaleza, $C E$ 60430-140, Brasil. carllosalencar@hotmail.com
}

\begin{abstract}
Chronic sequelae and disabilities are one of the main problems in leprosy. The current study aimed to estimate the prevalence of disabilities in leprosy patients after successfully completing multidrug therapy in Araguaina, Tocantins State, Brazil. This was a cross-sectional study including 282 cases diagnosed from 2004 to 2009. The degrees of disability at diagnosis and at discharge from treatment were collected from medical records and the National Information System for Notifiable Diseases (SINAN). A simplified neurological workup was performed after discharge from treatment. The prevalence of disabilities at diagnosis was 29.4\%, and $8.9 \%$ of then was grade II. Between diagnosis and discharge, the degree of physical disability worsened in $25 \%$ of cases. At diagnosis, the proportion of deformities was significantly higher in men (RR = 1.7; 95\%CI: 1.23-2.37). There was a significant association between disability and multibacillary disease $(p<0.001)$ and occurrence of reactions $(p<0.001)$. The data show that after discharge from multidrug therapy, in order to prevent chronic sequelae and functional limitations, continuous monitoring is still needed for individuals that have been recorded as cured and thus deleted from the records.
\end{abstract}

Disabled Persons; Leprosy; Communicable Diseases

\section{Resumo}

Danos neurais contribuem para a incapacidade física na hanseníase. O objetivo foi estimar a prevalência de indivíduos com incapacidade física após a alta de poliquimioterapia da hanseníase em Araguaína, Tocantins, Brasil. Estudo transversal com 282 casos novos de 2004 a 2009. O grau de incapacidade no diagnóstico e na cura foi coletado de prontuários e do Sistema de Informação de Agravos de Notificação. No pós-alta usou-se o formulário de avaliação neurológica simplificada. A prevalência de incapacidade foi de 29,4\%, sendo 8,9\% grau 2. Houve associação entre incapacidades físicas com multibacilares $(p<0,001)$ e com episódios reacionais $(p<0,001)$. No diagnóstico, a ocorrência de deformidades foi 1,7 vez maior nos homens (IC95\%: 1,23-2,37). Entre o diagnóstico e a alta houve piora do grau de incapacidade e esta piora foi maior após a alta: 25\%. A piora do grau de incapacidade foi mais expressiva após a alta, apresentando associação com multibacilares e episódios reacionais. É preciso monitoramento contínuo de casos em alta, para prevenir sequelas e limitação funcional.

Pessoas com Deficiência; Hanseníase; Doenças Transmissíveis 


\section{Introdução}

Ainda no século XXI, a detecção de casos novos de hanseníase permanece elevada no mundo, com cerca de 250 mil casos novos registrados a cada ano. Em torno de 15 milhões de pessoas foram tratadas com poliquimioterapia desde a sua implementação na década de 1980 até meados de 2010, e destas, aproximadamente 2 milhões estão desenvolvendo algum tipo de incapacidade, principalmente na faixa etária economicamente ativa 1 . É uma importante morbidade, por levar a incapacidades, deformidades físicas, preconceito e estigma, apesar de não se apresentar como causa básica frequente de óbito 2 .

Cerca de $20 \%$ das pessoas atingidas pela hanseníase podem sofrer de incapacidades ou problemas psicossociais, com necessidade de algum tipo de apoio e/ou reabilitação 3,4.

No Brasil, mesmo com todos os esforços e avanços empreendidos na integração do controle da hanseníase na rede de atenção à saúde, esta doença é ainda considerada um problema de saúde pública 5,6. O Brasil foi o segundo país com o maior número de casos em 2012 e apresentou aproximadamente 93\% dos casos das Américas 7,8 . No país, foram diagnosticados 2.165 $(7,1 \%)$ casos novos com grau 2 de incapacidade no ano de 20116.

Nesse ano, o Estado do Tocantins foi classificado como o segundo estado brasileiro com maior coeficiente de detecção para hanseníase, em níveis hiperendêmicos de 72,14 casos novos/100 mil habitantes em 2011 6, sendo que a média de casos com incapacidades de graus 1 e 2 no período de 2001 a 2010 foi de $18,3 \%$ e $5,1 \%$, respectivamente. Por sua vez, o Município de Araguaína no norte do Estado do Tocantins está inserido em uma área de aglomeração de casos e representa alto risco para a transmissão da hanseníase 5,9. No período de 2001 a 2010, o coeficiente médio de casos com grau 2 por 100 mil habitantes foi de 4,2 e a média da proporção de casos com grau 2 entre os avaliados foi de 3,9; havendo uma tendência de estabilidade desses indicadores.

O acompanhamento sistemático após a alta de pessoas que foram diagnosticadas com hanseníase, apresentando ou não incapacidades físicas, possibilitaria uma melhor vigilância em relação às complicações crônicas da doença. Nessa perspectiva, o Ministério da Saúde preconiza que "o(a) paciente deve ser orientado(a) para o retorno pós-alta por cura de acordo com as suas necessidades", e fica excluído do registro ativo de casos a serem acompanhados 10 .

No contexto de ser uma população mais negligenciada pelos serviços de saúde comparativa- mente àquela que está em tratamento, buscou-se neste estudo estimar a prevalência de indivíduos com incapacidade física em hanseníase após alta de poliquimioterapia no Município de Araguaína. A proposta é subsidiar e aprimorar a qualificação da atenção integral às pessoas atingidas pela hanseníase e auxiliar o planejamento de ações de controle no Sistema Único de Saúde (SUS), na perspectiva da longitudinalidade do cuidado de uma condição crônica.

\section{Material e métodos}

Este estudo faz parte de um projeto maior da Universidade Federal do Ceará denominado INTEGRAHANS - MAPATOPI, baseado em uma abordagem integrada de estudos relativos aos padrões epidemiológicos, clínicos, psicossociais e operacionais da hanseníase nos estados do Maranhão, Pará, Tocantins e Piauí, com o apoio financeiro do Departamento de Ciência e Tecnologia (DECIT) do Ministério da Saúde.

É um estudo transversal conduzido no Município de Araguaína, extremo norte do Estado do Tocantins. O município está situado na região da Amazônia Legal. Sua população estimada em 2010 foi de aproximadamente 150 mil habitantes, distribuída em uma área territorial de $277.620,9 \mathrm{~km}^{2}$.

A população de estudo foi constituída por todos os casos novos com 15 anos de idade ou mais, que receberam alta por cura da hanseníase no período de janeiro de 2004 a dezembro de 2009, totalizando 693 casos diagnosticados no município. Fizeram parte do estudo 282 pessoas diagnosticadas, tratadas e residentes em Araguaína amostradas por conveniência. Conforme os critérios do Ministério da Saúde, alta por cura é definida como o período após o término do tratamento da poliquimioterapia ${ }^{10}$. Os participantes responderam a uma entrevista padronizada e as informações complementares foram obtidas com base nos dados do Sistema de Informação de Agravos de Notificação (SINAN) e dos respectivos prontuários médicos das unidades básicas de saúde onde foram tratados e acompanhados. Investigaram-se variáveis sociodemográficas e clínicas como sexo, idade, escolaridade, raça/ cor, ocupação, zona de residência, classificação operacional, forma clínica, grau de incapacidade física no diagnóstico, alta e pós-alta e ocorrência de reações hansênicas, tipo de atendimento no pós-alta.

Depois da entrevista, foi realizado um exame clínico por profissionais de referência treinados para identificação da limitação funcional, utilizando-se o instrumento de avaliação 
simplificada das funções neurais e de suas complicações 11. A avaliação da magnitude das incapacidades foi realizada por meio da definição do maior grau de incapacidade, conforme preconizado pelo Ministério da Saúde 10,12. O grau de incapacidade no diagnóstico e na cura foi obtido baseando-se nos registros de prontuários dos indivíduos e do SINAN. Comparou-se o grau de incapacidade física entre o momento do diagnóstico em relação à alta e do momento da alta em relação ao pós-alta. A presença de reações foi identificada com base nos registros dos prontuários, tanto no diagnóstico quanto na alta e no período pós-alta. Para análise, os casos foram classificados como melhora, piora ou permanência do grau. Foi considerada como piora a perda de sensibilidade e/ou surgimento de alterações ao longo do período do tratamento até o momento após a alta. A incapacidade física foi classificada por meio do maior grau observado ao exame: grau 0 (nenhuma incapacidade relacionada à hanseníase), grau 1 (diminuição ou perda da sensibilidade) e grau 2 (presença de incapacidades e deformidades visíveis) $10,11,12$.

Para análise dos dados utilizou-se o programa Stata 11 (Stata Corp., College Station, Estados Unidos). Para a análise, além da abordagem descritiva em tabelas e gráficos, foi utilizado o teste de qui-quadrado de Pearson mais o cálculo das razões de prevalência (RP) relativas à piora do grau de incapacidade ou de seu surgimento, com intervalos de 95\% de confiança (IC95\%) e nível de $5 \%$ de significância.

O trabalho foi aprovado pelo Comitê de Ética em Pesquisa do Centro Universitário Luterano de Palmas - Tocantins (protocolo no 28/2009/CEP/ ULBRA). A coleta de dados foi realizada após o consentimento livre por escrito do participante (ou do seu representante legal) depois do esclarecimento dos objetivos da pesquisa.

\section{Resultados}

Entre as 693 pessoas previstas inicialmente, foram avaliadas 282 (40,7\% da população alvo). Os 411 (59,3\%) indivíduos não incluídos no estudo tiveram como justificativa para perda: não localização e/ou mudança de endereço 287 (69,8\%), não comparecimento $69(16,8 \%)$, recusa $31(7,5 \%)$ e outros motivos 25 (6\%). O sexo masculino foi predominante com $145(51,4 \%)$ indivíduos. A média de idade foi de 45,8 anos, com amplitude de 15 a 85 anos. Em relação à escolaridade, havia 110 (39\%) pessoas com menos de 5 anos de estudos, 64 (22,7\%) tinham de 5-9 anos e 108 (38,3\%) relataram ter mais de 10 anos. A cor parda foi a mais referida, 170 (60,2\%) pessoas, e 267 (94,7\%) residiam na zona urbana.

De acordo com a classificação brasileira de ocupações/profissões, o grupo mais frequente foi de trabalhadores de serviços, vendedores do comércio em lojas e mercados - 69 (39,4\%), técnicos de nível médio - $50(30,8 \%)$ e trabalhadores agropecuários, florestais, da caça e pesca - 18 (10,3\%). Trabalhadores de serviços administrativos e produção de bens e serviços industriais foram representados por $11(6,2 \%)$ indivíduos cada e $12(4,2 \%)$ foram classificados como outros. Os demais eram aposentados 39 $(13,8 \%)$, do lar 39 (13,8\%), estudantes 17 (6\%), beneficiados/pensionistas $12(4,2 \%)$ e desempregados $4(1,4 \%)$.

Houve uma maior frequência de casos paucibacilares $(170 ; 60,3 \%)$ e da forma clínica indeterminada $(96 ; 34 \%)$. No período pós-alta, a ocorrência de incapacidades físicas foi mais frequente com 58 (20,6\%) casos de grau 1 e 25 (8,9\%) de grau 2 (Tabela 1). O mesmo ocorreu para os episódios reacionais com 99 (35,1\%) casos.

Entre as 282 pessoas acometidas pela hanseníase, um total de 56 (19,8\%) indivíduos apresentou reação hansênica no momento do diagnóstico. Desses, 53 (94,6\%) permaneceram com reação hansênica até o final do tratamento, e outros 35 $(12,4 \%)$ desencadearam novo episódio durante o tratamento. Dos 88 (31,2\%) casos identificados com episódios reacionais no momento da alta, $72(25,5 \%)$ permaneceram em reação após o final do tratamento, enquanto que 27 (9,6\%) novos episódios reacionais surgiram neste período. Por fim, 99 (35,1\%) apresentaram reação no período do pós-alta (Tabela 1).

Os episódios reacionais quando relacionados às formas clínicas foram mais ocorrentes principalmente no período pós-alta. Na forma clínica indeterminada a ocorrência foi de $7(2,4 \%)$ casos no diagnóstico, $13(4,6 \%)$ na alta e $18(6,4 \%)$ no pós-alta. Na forma clínica tuberculoide as reações ocorreram em 8 (2,8\%) casos no diagnóstico, 14 (5\%) na alta e mais 14 (5\%) no pós-alta. A forma clínica dimorfa foi responsável pela maior ocorrência dos episódios reacionais com 22 $(7,8 \%)$ casos no diagnóstico, 34 (12\%) na alta e 37 (13\%) no pós-alta. Na forma clínica virchowiana houve $19(6,7 \%)$ casos com episódios reacionais no diagnóstico, 27 (9,5\%) na alta e $30(10,6 \%)$ no pós-alta.

Após a conclusão da poliquimioterapia, a frequência de episódios reacionais em um período de tempo de menos de 6 meses foi de 9 (9\%) casos entre 6 meses e 1 ano, de 6 (6\%) casos a partir de 1 ano a 2,5 anos , de $40(40,4 \%)$ casos, de 37 $(39,3 \%)$ casos entre 2,5 a 5 anos e de 7 (7\%) casos após 5 anos de alta. 
Caracterização clínica das pessoas em pós-alta de hanseníase no período de janeiro de 2004 a dezembro de 2009. Araguaína, Tocantins, Brasil.

\begin{tabular}{|c|c|c|}
\hline Variáveis & $n(N=282)$ & $\%$ \\
\hline \multicolumn{3}{|c|}{ Classificação operacional } \\
\hline Paucibacilar & 170 & 60,3 \\
\hline Multibacilar & 112 & 39,7 \\
\hline \multicolumn{3}{|l|}{ Forma clínica } \\
\hline Indeterminada & 96 & 34,0 \\
\hline Tuberculoide & 74 & 26,2 \\
\hline Dimorfa & 75 & 26,6 \\
\hline Virchowiana & 37 & 13,1 \\
\hline \multicolumn{3}{|c|}{ Grau de incapacidade no diagnóstico } \\
\hline Grau 0 & 238 & 84,4 \\
\hline Grau 1 & 37 & 13,1 \\
\hline Grau 2 & 7 & 2,5 \\
\hline \multicolumn{3}{|c|}{ Grau de incapacidade na alta } \\
\hline Grau 0 & 240 & 85,1 \\
\hline Grau 1 & 26 & 9,2 \\
\hline Grau 2 & 13 & 4,6 \\
\hline Ignorado & 3 & 1,1 \\
\hline \multicolumn{3}{|c|}{ Grau de incapacidade no período pós-alta * } \\
\hline Grau 0 & 199 & 70,5 \\
\hline Grau 1 & 58 & 20,6 \\
\hline Grau 2 & 25 & 8,9 \\
\hline
\end{tabular}

* Três registros não apresentavam informações quanto ao grau de incapacidade no momento da alta.

A frequência observada de incapacidades com graus 1 ou 2 na faixa etária de 15-20 anos foi de $2(0,7 \%)$ casos, na de 20-35 anos de $11(3,9 \%)$ casos, na de $35-50$ anos de 19 (6,7\%), na de 50-65 anos de $27(9,6 \%)$ e na de 65 ou mais anos de 27 $(8,5 \%)$ casos.

Observou-se nos homens uma estimativa de prevalência 1,7 vez maior para deformidades físicas no diagnóstico (IC95\%: 1,23-2,37). Na alta, a prevalência de incapacidades foi semelhante entre os sexos (IC95\%: 0,63-1,79). No pós-alta, a estimativa de prevalência foi 1,6 maior para a incapacidade de grau 2 nos homens (IC95\%: 1,23-2,09).

Houve maior estimativa de prevalência de incapacidades físicas nas pessoas com forma clínica multibacilar em relação à paucibacilar. Essa associação manteve-se estatisticamente significante no momento do diagnóstico, na alta da poliquimioterapia e no pós-alta, sendo 3,1 vezes maior nas pessoas com incapacidade de grau 2 (Tabela 2).

A estimativa da prevalência de incapacidades físicas também foi maior entre as pessoas que apresentaram episódios reacionais, quando comparadas àquelas que não apresentaram nos momentos da avaliação. As incapacidades físicas de grau 2 foram 3,6 vezes mais frequentes no momento do diagnóstico e 2,5 vezes mais frequentes no pós-alta (Tabela 3).

A maioria das pessoas com incapacidade física de grau 1 ou 2 não apresentou episódios reacionais nos momentos do diagnóstico (26 casos; $9,2 \%$ ), da alta ( 16 casos; $5,7 \%$ ) e do pós-alta (37 casos; $13,1 \%$ )

A comparação do grau de incapacidade física entre o momento do diagnóstico e da alta da poliquimioterapia evidenciou que houve piora do grau em $19(6,7 \%)$ pessoas, e outras $19(6,7 \%)$ permaneceram com o mesmo grau, outras $25(8,5 \%)$ tiveram melhora das incapacidades de grau $1 \mathrm{e} /$ ou 2 para grau 0 . Três (1\%) pessoas não tinham registro do grau de incapacidade na alta.

A análise da progressão do grau de incapacidade física entre o momento da alta e do pósalta evidenciou a piora do grau em $71(25,1 \%)$ pessoas, $11(3,9 \%)$ permaneceram com o mesmo grau e 18 (6,3\%) melhoram. A Tabela 4 apresenta a síntese da progressão do grau de incapacidade física. 
Tabela 2

Grau de incapacidade física segundo forma de classificação operacional da hanseníase no período 2004 a 2009. Araguaína, Tocantins, Brasil.

\begin{tabular}{|c|c|c|c|c|c|c|}
\hline \multirow[t]{2}{*}{ Grau de incapacidade física } & \multirow{2}{*}{$\begin{array}{c}\text { Total } \\
\mathbf{n}\end{array}$} & \multicolumn{2}{|c|}{ Multibacilar } & \multirow[t]{2}{*}{ RP } & \multirow[t]{2}{*}{ IC95\% } & \multirow[t]{2}{*}{ Valor de $p$} \\
\hline & & $\mathrm{n}$ & $\%$ & & & \\
\hline \multicolumn{7}{|l|}{ Diagnóstico } \\
\hline 0 & 238 & 79 & 33,2 & 1,00 & - & 0,000 \\
\hline 1 & 37 & 28 & 75,7 & 2,27 & $1,76-2,94$ & \\
\hline 2 & 7 & 5 & 71,4 & 2,15 & $1,30-3,55$ & \\
\hline \multicolumn{7}{|l|}{ Alta } \\
\hline 0 & 240 & 81 & 33,8 & 1,00 & - & 0,000 \\
\hline 1 & 26 & 22 & 84,6 & 2,50 & $1,96-3,19$ & \\
\hline 2 & 13 & 7 & 53,8 & 1,59 & $0,93-2,72$ & \\
\hline \multicolumn{7}{|l|}{ Pós-alta } \\
\hline 0 & 199 & 59 & 29,6 & 1,00 & - & 0,000 \\
\hline 1 & 58 & 30 & 51,7 & 1,74 & $1,25-2,42$ & \\
\hline 2 & 25 & 23 & 92,0 & 3,10 & $2,43-3,95$ & \\
\hline
\end{tabular}

IC95\%: intervalo de 95\% de confiança; RP: razão de prevalência.

Tabela 3

Grau de incapacidade física segundo presença de episódios reacionais da hanseníase no período 2004 a 2009. Araguaína, Tocantins, Brasil.

\begin{tabular}{|c|c|c|c|c|c|c|}
\hline \multirow[t]{2}{*}{ Grau de incapacidade física } & \multirow{2}{*}{$\begin{array}{c}\text { Total } \\
\mathbf{n}\end{array}$} & \multicolumn{2}{|c|}{ Com episódio reacional } & \multirow[t]{2}{*}{$\mathrm{RP}$} & \multirow[t]{2}{*}{ IC95\% } & \multirow[t]{2}{*}{ Valor de $\mathrm{p}$} \\
\hline & & $\mathbf{n}$ & $\%$ & & & \\
\hline \multicolumn{7}{|l|}{ Diagnóstico } \\
\hline 0 & 238 & 38 & 16,0 & 1,00 & - & 0,000 \\
\hline 1 & 37 & 14 & 37,8 & 2,36 & $1,42-3,90$ & \\
\hline 2 & 7 & 4 & 57,1 & 3,57 & $1,76-7,24$ & \\
\hline \multicolumn{7}{|l|}{ Alta * } \\
\hline 0 & 240 & 65 & 27,1 & 1,00 & - & 0,000 \\
\hline 1 & 26 & 18 & 69,2 & 2,55 & $1,83-3,55$ & \\
\hline 2 & 13 & 5 & 38,5 & 1,42 & $0,69-2,91$ & \\
\hline \multicolumn{7}{|l|}{ Pós-alta } \\
\hline 0 & 199 & 53 & 29,6 & 1,00 & - & 0,000 \\
\hline 1 & 58 & 29 & 50,0 & 1,87 & $1,32-2,65$ & \\
\hline 2 & 25 & 17 & 68,0 & 2,55 & $1,79-3,63$ & \\
\hline
\end{tabular}

IC95\%: intervalo de 95\% de confiança; RP: razão de prevalência.

* Não foram encontrados três registros na alta.

Entre as 282 pessoas avaliadas (correspondendo a 564 olhos, mãos e pés), identificaram-se incapacidades físicas em 110 (19,5\%) pés, sendo este o seguimento mais afetado, seguido pelas mãos 30 (5,3\%) e pelos olhos 29 (5,1\%).

Trinta e duas (11,3\%) pessoas apresentaram incapacidade de grau 1 em ambos os pés, $9(3,2 \%)$ em ambos os olhos, $5(1,7 \%)$ pessoas apresentaram grau 1 para um pé e grau 2 para o outro, e 3 (1\%) pessoas tiveram grau 0 para um dos olhos e grau 2 para o olho oposto. Verificouse em 7 (2,5\%) casos grau 2 em ambas as mãos, ao passo que $8(2,8 \%)$ pessoas apresentaram grau 2 em ambos os pés, e 1 pessoa (0,3\%) com grau 2 bilateralmente nos olhos (Figura 1). 
Tabela 4

Comparação do grau de incapacidade física entre o momento do diagnóstico e da alta da poliquimioterapia e da alta ao pós-alta de hanseníase no período de 2004 a 2009. Araguaína, Tocantins, Brasil.

\begin{tabular}{|c|c|c|c|c|c|c|}
\hline \multirow[t]{3}{*}{ Diagnóstico } & \multicolumn{6}{|c|}{ Grau de incapacidade física } \\
\hline & \multicolumn{3}{|c|}{ Alta } & \multicolumn{3}{|c|}{ Pós-alta } \\
\hline & 0 & 1 & 2 & 0 & 1 & 2 \\
\hline 0 & $217(91,2 \%)$ & $10(4,2 \%)$ & $8(3,4 \%)$ & $179(74,6 \%)$ & $51(21,2 \%)$ & $10(4,2 \%)$ \\
\hline 1 & $21(56,8 \%)$ & $15(40,5 \%)$ & $1(2,7 \%)$ & $10(38,4 \%)$ & $6(23,1 \%)$ & $10(38,5 \%)$ \\
\hline 2 & $2(28,6 \%)$ & $1(14,6 \%)$ & $4(57,1 \%)$ & $7(53,8 \%)$ & $1(7,7 \%)$ & $5(38,5 \%)$ \\
\hline
\end{tabular}

Figura 1

Incapacidade física por hanseníase segundo segmento acometido (olho, mão e pé) em pessoas no momento do pós-alta, no período de janeiro de 2004 a dezembro de 2009. Araguaína, Tocantins, Brasil.

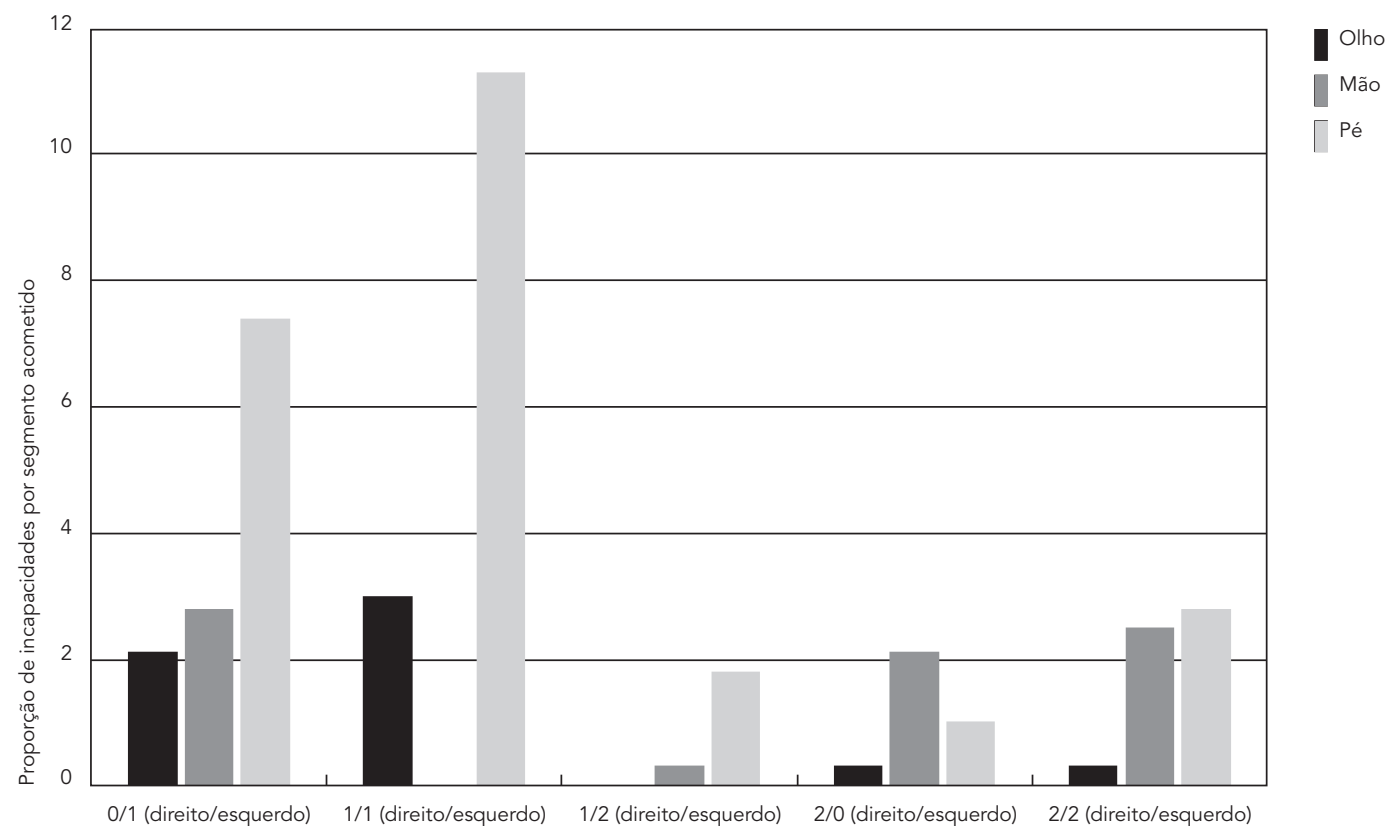

No pós-alta, as pessoas que retornaram aos serviços de saúde para acompanhamento devido a episódios reacionais foram atendidas pelo profissional médico em $100 \%$ dos casos, $47(47,4 \%)$ pela fisioterapia e $17(17,1 \%)$ pela enfermagem. Profissionais das áreas de psicologia, odontologia e serviço social atenderam um caso cada $(1,1 \%$, cada).

No período desta avaliação, 73 (25,9\%) pessoas foram identificadas com complicações crô- nicas da hanseníase após a cura e foram encaminhadas para diferentes serviços de saúde e especialidades, sendo $33(45,2 \%)$ para a dermatologia, $24(32,9)$ para a clínica geral, $12(16,4 \%)$ para a sapataria, $7(9,5 \%)$ para a fisioterapia, $3(4,1 \%)$ para a psicologia, $3(4,1 \%)$ para a oftalmologia e 1 $(1,4 \%)$ para a cirurgia. 


\section{Discussão}

No nosso estudo, após a alta da poliquimioterapia, a estimativa de prevalência de incapacidades (cerca de 30\%) e das incapacidades de grau 2, deformidades físicas visíveis (cerca de 9\%), apresentou valores médios segundo os parâmetros epidemiológicos do Ministério da Saúde. Provavelmente isso ocorreu pela inadequação do seguimento clínico após a saída do registro ativo. Por outro lado, essa estimativa foi considerada baixa no momento diagnóstico e da alta da poliquimioterapia, indicando focalização da atenção durante o tratamento específico. Na série histórica de 2004 a 2009, os indicadores epidemiológicos de Araguaína relacionados à proporção de pessoas avaliadas quanto ao grau de incapacidade física no diagnóstico e na cura foram considerados satisfatórios de acordo com critérios do Ministério da Saúde 13.

Reconhece-se que o grau de incapacidade está relacionado diretamente com o tempo de evolução da doença 14, o que reforça a necessidade de estruturação de seguimento qualificado, na perspectiva da integralidade de todos os casos diagnosticados durante e após a poliquimioterapia. O conhecimento dessa população em diferentes perspectivas amplia a possibilidade de qualificar essa atenção. O maior contingente de pessoas no pós-alta era de trabalhadores ativos com ocupações que requeriam grande esforço físico e habilidades funcionais plenas, que comumente são afetadas pelas complicações decorrentes da hanseníase. O comprometimento sensitivo é mais comum em trabalhadores braçais 15 . As pessoas acometidas pela hanseníase podem sofrer prejuízo na sua capacidade de trabalho por ocasião das deformidades e, consequentemente, no autossustento e da família, gerando repercussões de ordem psicológica, social e física 16,17 .

Essa população atingiu uma alta proporção de pessoas com idade economicamente ativa como observado em outros estudos 18. A estimativa da prevalência de deformidades no pós-alta variou com a idade, corroborando com a literatura 19 , o que compromete potencialmente a realização de atividades diárias com repercussões físicas, sociais e econômicas.

A maioria apresentava menos de cinco anos de estudos, situação encontrada em outros trabalhos 20,21,22,23,24. Na Região Nordeste do país, a baixa escolaridade esteve associada às incapacidades físicas 22,25, na Índia a situação também foi condizente com esta realidade 26 , o que reflete a condição social e econômica dessa população. Essa situação pode ampliar a vulnerabilidade dessas pessoas, podendo dificultar a compreen- são, adoção de tratamento e de medidas para o autocuidado.

O padrão observado em relação à cor e zona de residência está consistente com o relatado na literatura 18,20,27. O fato da quase totalidade residir na zona urbana proporciona, pelo menos em tese, melhores condições para acesso aos serviços de saúde. Diante da realidade demográfica tocantinense referente à raça/cor, os pardos de fato representam o contingente populacional mais frequente, fato este refletido no estudo (Instituto Brasileiro de Geografia e Estatística. Estados@.http://www.ibge.gov.br/estadosat/perfil. php?sigla=to, acessado em 24/Nov/2011).

Os homens foram os mais atingidos pelas deformidades, classificados em maior proporção como multibacilares, mas sem significância estatística. As deformidades são mais significativas para o sexo masculino, o que confirma uma maior proporção de diagnóstico tardio em homens 15,28, além de aspectos relativos ao autocuidado nesta população. A maior ocorrência de casos no sexo masculino foi observada em outros cenários nas várias regiões do Brasil 6,22,28. Os homens apresentam os maiores coeficientes de detecção de casos de hanseníase e o risco de exposição nesta população é amplamente reconhecido 1,5,18,28.

As formas clínicas indeterminada e dimorfa foram predominantes. Mas houve maior percentual dos casos paucibacilares, acompanhando o padrão clínico epidemiológico nacional e estadual 6,24,29,30.

A doença apresenta maior detecção entre os homens, porém, a razão entre os sexos diminuiu ao longo dos últimos anos e os efeitos degenerativos nas mulheres não ocorrem com a mesma intensidade que nos homens devido a uma maior procura destas pelos serviços de saúde 28,31.

A estimativa da prevalência de incapacidades físicas mostrou predomínio da incapacidade de grau 1, consistente também com dados oficiais e de estudos anteriores 6,18,19,20.

Neste trabalho, o maior contingente dos episódios reacionais ocorreu no primeiro ano após o diagnóstico, sendo mais frequente no período pós-alta, visto que do total de pessoas que apresentaram estes episódios após a alta, pouco menos de um terço não apresentou este evento durante o tratamento. Esses achados evidenciam uma correlação entre a ocorrência de episódios reacionais durante o tratamento e após a alta da poliquimioterapia, situação condizente com o observado na literatura 19,32.

As formas clínicas multibacilares foram responsáveis pela maioria dos episódios reacionais, entretanto houve destaque no número de pessoas tratadas com episódios reacionais e que foram classificadas na forma clínica indeterminada, 
em todos os momentos da avaliação. Supõe-se que essa situação tenha ocorrido por um provável erro na classificação da forma clínica no diagnóstico.

Reconhece-se que as os casos multibacilares representam fatores de risco para o desenvolvimento de episódios reacionais. Além disso, a forma indeterminada também pode evoluir durante o tratamento para formas multibacilares, explicando a ocorrência de reações com a forma indeterminada 21 .

$\mathrm{O}$ fator que pode condicionar um maior contingente de deformidades no pós-alta é a falta de um adequado monitoramento dos quadros reacionais pela rede de serviços de saúde nesse período. As pessoas que apresentam episódio reacional após o término do tratamento são mais propensas a terem deformidades físicas, pelo fato de já estarem fora do registro e não serem mais acompanhadas. Isso pode levá-las a não considerar sinais e sintomas de possíveis complicações, com a procura tardia dos serviços de saúde. O monitoramento pós-alta deve fazer parte da rotina dos serviços de saúde. Uma avaliação realizada em Minas Gerais, após um tempo médio de 18 anos do início do tratamento, mostrou que $10,2 \%$ das pessoas curadas apresentaram episódios reacionais caracterizando o longo período de evolução desta condição crônica 23 .

Aproximadamente um terço das pessoas avaliadas apresentava alguma incapacidade física, sem considerar a existência de episódio reacional relacionado em qualquer dos momentos de avaliação. Esse fato leva a acreditar que a avaliação clínica das pessoas acometidas pela hanseníase não vinha sendo realizada de forma sistematizada, e/ou as informações relativas à presença de episódio reacional não foram registradas nos prontuários em sua totalidade. $\mathrm{O}$ comprometimento da função neural comumente ocorre durante ou após a poliquimioterapia e é fator determinante para levar à incapacidade ou à deformidade, sendo que a forma multibacilar aumenta o risco em $65 \%$ dos casos 14 .

A falta de protocolos padronizados que direcionem a classificação e o manejo dos episódios reacionais, bem como suas possíveis repercussões na progressão do grau de incapacidade física pelos serviços de saúde é um dos fatores que dificulta o conhecimento da situação clínica das pessoas afetadas 33 . Na demanda pós-alta, estudo em Minas Gerais identificou $67,7 \%$ pessoas com grau 0 de incapacidade que retornaram aos serviços de saúde por reação e/ou neurite 34 .

$\mathrm{O}$ maior percentual de deformidades encontradas após a alta pode ser reflexo da descontinuidade da assistência depois da exclusão do registro ativo. Porém, aproximadamente $20 \%$ de todos os casos novos apresentam incapacidades no momento do diagnóstico e outros 15\% irão desenvolvê-las mesmo que todas as ações de saúde sejam realizadas adequadamente, seja no tratamento das neurites, dos estados reacionais e mesmo com a poliquimioterapia 35 . Uma elevada estimativa da prevalência de incapacidade grau 2 oscilando entre $17 \%$ a $50 \%$ após a alta foi verificada em estudo em países asiáticos 3 .

Os indicadores de prevalência de deformidades físicas visíveis (grau 2) no Brasil apresentam menores proporções quando comparados com a realidade de países asiáticos; isto pode ser explicado pela ampliação da assistência na atenção primária em saúde, refletindo melhoria dos serviços de controle da hanseníase no diagnóstico precoce e tratamento oportuno. A estimativa da prevalência média de incapacidade grau 2 foi semelhante a outros estudos, 8,7\% em Uberlândia (Minas Gerais) 19, 10\% em Belo Horizonte (Minas Gerais) 36, 10\% em Várzea Grande (Mato Grosso) 15 e de 7\% em Buriticupu (Maranhão) 20.

As variáveis associadas às incapacidades físicas no momento do diagnóstico, alta e após a alta foram forma clínica e ocorrência de episódios reacionais. A estimativa da prevalência de incapacidades foi significativamente superior nos casos multibacilares e que tiveram episódios reacionais, consistente com o verificado na literatura ${ }^{37}$. A estimativa de prevalência de incapacidades de grau 2 foi maior no momento pós-alta (3,5 vezes) nos casos multibacilares quando comparada com os paucibacilares. Nos casos com episódios reacionais, a proporção de incapacidades de grau 2 foi maior no diagnóstico (3,5 vezes), seguida pelo momento pós-alta (2,5 vezes).

As formas avançadas da doença, bem como os episódios reacionais, mostraram-se importantes preditores para deformidades físicas, reforçando a necessidade de melhor qualidade nas medidas para o controle da doença. Essas incluem diagnóstico precoce dos casos e das neuropatias, além de tratamento específico com $\mathrm{o}$ intuito de minimizar as incapacidades físicas e sequelas associadas 14,38 .

Identificaram-se situações em que pessoas com grau 0 de incapacidade passaram para os graus 1 ou 2 no período entre o diagnóstico e a alta, mas esta progressão foi mais expressiva entre o momento da alta e o da pós-alta. Essa situação estava possivelmente condicionada pelo diagnóstico tardio das neuropatias, bem como pela fragilidade das ações de prevenção de incapacidades instituídas que deveriam ser adotadas em um momento oportuno. Os casos que saem do registro ativo com alta por cura, deveriam ser monitorados pelos serviços de saúde para a manutenção desta condição, visto que este indica- 
dor sinaliza uma boa qualidade da atenção nos serviços de saúde.

Em Mato Grosso, aproximadamente 50\% dos pacientes tiveram piora da sensibilidade entre alta e o pós-alta ${ }^{15}$. Em outro estudo, realizado em Rondônia, foi visto que entre os casos com incapacidade de grau 0 no início do tratamento, $5 \%$ paucibacilares e $20 \%$ multibacilares pioraram o grau após o tratamento 39 .

Os dados de uma coorte de casos de hanseníase com incapacidades em Belo Horizonte evidenciaram uma redução de incapacidades em $15 \%$ dos casos, entre o diagnóstico e a alta, sendo que os fatores preditivos para esta boa evolução foram diagnóstico precoce das neuropatias, adequada corticoterapia e técnicas de prevenção de incapacidades aplicadas durante o tratamento 36 . Em Fortaleza, mesmo com o baixo percentual de pessoas avaliadas quanto ao grau de incapacidade no diagnóstico e na alta, identificou-se uma fração importante de deformidades em menores de 15 anos, revelando a manutenção da endemia e diagnóstico tardio 40.

O Programa Nacional de Controle da Hanseníase recomenda o monitoramento de pessoas apresentando episódios reacionais, visto que estas podem evoluir com piora das funções neurais e complicações 12. Contudo, a avaliação sistemática das pessoas em pós-alta de hanseníase deveria fazer parte da rotina da rede de serviços de saúde local, pois o adequado manejo destas pessoas é o que determinará, em grande parte, a prevenção de complicações, redução de custos com reabilitação e melhora da qualidade de vida.

$\mathrm{Na}$ análise do grau de incapacidade por seguimento acometido, os pés foram a estrutura mais afetada, seguidos pelas mãos e olhos. Assim, há uma clara necessidade de programação de ações que focalizem a longitudinalidade do cuidado, incluindo intervenções reabilitadoras e reparadoras pelos serviços de saúde. Isso reforça a importância da avaliação precoce, nas diferentes fases de tratamento da doença, bem como após a alta, focando a orientação no autocuidado e na indicação do uso de calçados especiais para prevenir lesões que possam limitar os participantes de atividades da vida diária.

Os casos avaliados muitas vezes vinham apresentando comprometimento bilateral ou estavam progredindo para este quadro, o que configura gravidade máxima da incapacidade pela possibilidade de uma deformidade bilateral e consequentemente invalidez. Nesse aspecto, observaram-se pessoas evoluindo para a cegueira bilateral, deformidades em ambos os pés e/ou mãos, apresentando necessidade máxima de intervenções de prevenção de incapacidades e mesmo de reabilitação. Essa conduta é amplificada para aqueles que já se encontram cegos, com os dois seguimentos de pés e mãos deformados pelas complicações da hanseníase e que não estão sendo assistidos pelos serviços de saúde por estarem curados do ponto de vista microbiológico.

Tais situações geram potencialmente um prejuízo à funcionalidade corporal e a perda da qualidade de vida das pessoas acometidas, podendo acarretar vários estigmas, bem como custos individuais e para o setor público.

Em relação à demanda pós-alta, as pessoas retornaram aos serviços de saúde para atendimento em consequência de episódios reacionais, e o profissional médico foi o responsável por todos os atendimentos, resultado condizente com uma avaliação realizada no Ceará ${ }^{18}$. O baixo número de registros de atendimentos por profissionais de outras categorias aponta para um caráter secundário das ações de prevenção de incapacidades e reabilitação nos serviços de saúde, comprometendo a longitudinalidade do cuidado.

Pessoas em alta, sem acompanhamento, foram encaminhadas para diferentes serviços e atendimentos e isto gerou uma grande demanda concentrada nos serviços locais de saúde, resultado de uma triagem clínica recomendada, que corrobora com outro achado 18.

\section{Limitação do estudo}

A principal limitação deste estudo se deu pela não localização e/ou mudança de endereço de $69,8 \%$ do total de pessoas em pós-alta de hanseníase, e ainda pelo não comparecimento de $16,8 \%$. Considera-se limitação também o preenchimento algumas vezes inadequado das fichas de notificação do SINAN, bem como de lacunas nas informações nos prontuários. A falta ou erro de informações nos prontuários foram questões observadas e que carecem de investigação para esclarecimento dos fatores que influenciam na inadequação do diagnóstico e monitoramento dos casos após a alta da poliquimioterapia.

\section{Lacunas e/ou necessidade de outros estudos}

Visto a realidade encontrada, faz-se necessária a padronização no processo de atendimento no momento do pós-alta, pois o acompanhamento pós-alta está voltado basicamente para o tratamento de reações hansênicas, ainda distanciado da integralidade do cuidado SUS. 


\section{Conclusão}

A prevalência de incapacidade nessa população de estudo foi de $29,4 \%$, sendo $8,9 \%$ apresentando grau 2. Houve associação entre a presença de incapacidade com as formas clínicas da doença e episódios reacionais. Diagnosticou-se progres- são para piora do grau de incapacidade entre o momento da alta para o pós-alta em pessoas, mas a prevalência foi ainda maior entre o período da alta e do pós-alta com casos. Esses achados reforçam a necessidade de priorizar a atenção da hanseníase como condição crônica inserida efetivamente na rede de atenção do SUS.

\section{Resumen}

Daños neurales contribuyen a la incapacidad física en la hanseniasis. El objetivo fue estimar la prevalencia de individuos con incapacidad física, tras el alta de poliquimioterapia de la hanseniasis en Araguaína, Tocan tins, Brasil. Estudio transversal con 282 casos nuevos de 2004 a 2009. El grado de incapacidad en el diagnóstico y en la cura se recogió de historiales médicos y del sis tema de información de quejas por notificación. En el post alta se usó el formulario de evaluación neurológica simplificada. La prevalencia de incapacidad fue de un 29,4\%, siendo un 8,9\% grado 2. Hubo asociación entre incapacidades físicas con multibacilares $(p<0,001) y$ con episodios reaccionales $(p<0,001)$. En el diagnóstico la ocurrencia de deformidades fue un 1,7 veces mayor en los hombres (IC95\%: 1,23-2,37). Entre el diagnóstico y el alta hubo un empeoramiento del grado de incapacidad y ese empeoramiento fue mayor tras el alta: un $25 \%$. El empeoramiento del grado de incapacidad fue más expresivo tras el alta, presentando asociación con multibacilares y episodios reaccionales. Es preciso el monitoreo continuo de casos en alta, para prevenir secuelas y limitación funcional.

Personas con Discapacidad; Lepra; Enfermedades Transmisibles 


\section{Colaboradores}

L. D. Monteiro contribuiu na concepção do projeto, coleta, análise e interpretação dos dados, redação do artigo e aprovação final da versão a ser publicada. C. H. M. Alencar colaborou na concepção do projeto, análise e interpretação dos dados, revisão crítica relevante do conteúdo intelectual e aprovação final da versão a ser publicada. J. C. Barbosa e J. Heukelbach participaram da concepção do projeto, análise e interpretação dos dados, revisão crítica relevante do conteúdo intelectual, aprovação final da versão a ser publicada. K. P. Braga colaborou na concepção do projeto, coleta dos dados, redação do artigo e aprovação final da versão a ser publicada. M. D. Castro colaborou na concepção do projeto, redação do artigo e aprovação final da versão a ser publicada.

\section{Referências}

1. Rodrigues LC, Lockwood DNJ. Leprosy now: epidemiology, progress, challenges, and research gaps. Lancet Infect Dis 2011; 11:464-70.

2. Habbema J. Trends in leprosy case detection worldwide since 1985. Lepr Rev 2004;75:19-33.

3. Deepak S. Answering the rehabilitation needs of leprosy-affected persons in integrated setting through primary health care services and community-based rehabilitation. Indian J Lepr 2003; 75:127-42.

4. Monteiro LD. Padrões de comprometimento neural, limitação de atividade, participação social e fatores associados nas pessoas em pós-alta de hanseníase nos anos de 2004-2009, Araguaína - TO [Dissertação de Mestrado]. Fortaleza: Faculdade de Medicina, Universidade Federal do Ceará; 2012.

5. Penna MLF, Oliveira ML, Penna GO. The epidemiological behaviour of leprosy in Brazil. Lepr Rev 2009; 80:332-44.

6. Ministério da Saúde. Coeficientes de detecção geral de casos novos de hanseníase Brasil e estados. http://www. portal.saude.gov.br (acessado em 05/ Mai/2011).

7. World Helath Organization. Global leprosy situation, 2011. Geneva: World Helath Organization; 2011.

\section{Agradecimentos}

Agradecemos o apoio do CNPq; DECIT/MS; CAPES; Secretaria de Estado da Saúde do Tocantins; Secretaria Municipal de Saúde de Araguaína, Coordenação de Vigilância Epidemiológica e Atenção Básica de Araguaína; Leprosy Rielief Association (LRA); Fundação de Medicina Tropical do Tocantins e Hospital de Doenças Tropicais do Tocantins. À Maria de Jesus Freitas de Alencar, assessora técnica da Nederlandse Stichting voor Leprabestrijding (NHR Brasil).
8. World Helath Organization. Global leprosy situation, 2012. Geneva: World Helath Organization; 2012.

9. Alencar CH, Ramos Jr. AN, Santos ES, Richter J, Heukelbach J. Clusters of leprosy transmission and of late diagnosis in a highly endemic area in Brazil: focus on different spatial analysis approaches. Trop Med Int Health 2012; 17:518-25.

10. Ministério da Saúde. Portaria no 3.125, de 7 de outubro de 2010. Diário Oficial da União 2010; 15 out.

11. Departamento de Vigilância Epidemiológica, Secretaria de Vigilância à Saúde, Ministério da Saúde. Manual de prevenção de incapacidades. $3^{\text {a }}$ Ed. Brasília: Ministério da Saúde; 2008.

12. Departamento da Atenção Básica, Secretaria de Políticas de Saúde, Ministério da Saúde. Guia para o controle da hanseníase. Brasília: Ministério da Saúde; 2002.

13. Departamento de Vigilância Epidemiológica, Secretaria de Vigilância em Saúde, Ministério da Saúde. Hanseníase no Brasil: dados e indicadores selecionados. Brasília: Ministério da Saúde; 2009. 
14. Croft RP, Nicholls PG, Steyerberg EW, Richardus $\mathrm{JH}$, Smith WCS. A clinical prediction rule for nervefunction impairment in leprosy patients. Lancet 2000; 355:1603-6.

15. Ramos JMH, Souto FJD. Incapacidade pós-tratamento em pacientes hansenianos em Várzea Grande, Estado de Mato Grosso. Rev Soc Bras Med Trop 2010; 43:293-7.

16. Diffey B, Vaz M, Soares MJ, Jacob AJ, Piers LS. The effect of leprosy-induced deformity on the nutritional status of index cases and their household members in rural South India: a socio-economic perspective. Eur J Clin Nutr 2000; 54:643-9.

17. Teixeira MAG, Silveira VM, França ER. Características epidemiológicas e clínicas das reações hansênicas em indivíduos paucibacilares e multibacilares, atendidos em dois centros de referência para hanseníase, na Cidade de Recife, Estado de Pernambuco. Rev Soc Bras Med Trop 2010; 43:287-92.

18. Barbosa JC. Pós-alta em hanseníase no Ceará: olhares sobre políticas, rede de atenção à saúde, limitação funcional, de atividades e participação social das pessoas atingidas [Tese de Doutorado]. São Paulo: Faculdade de Saúde Pública, Universidade de São Paulo; 2009.

19. Rodrigues ALP, Almeida AP, Rodrigues BF, Pinheiro CA, Borges DS, Mendonça MLH, et al. Ocorrência de reações em pacientes pós-alta por cura de Hanseníase: subsídios para implementação de um programa de atenção específica. Hansenol Int 2000; 25:7-16.

20. Aquino DM, Caldas AJ, Silva AA, Costa JM. Perfil dos pacientes com hanseníase em área hiperendêmica da Amazônia do Maranhão, Brasil. Rev Soc Bras Med Trop 2003; 36:57-64.

21. Silva SF, Griep RH. Reação hansênica em pacientes portadores de hanseníase em centros de saúde da Área de Planejamento do Município do Rio de Janeiro. Hansenol Int 2007; 32:155-62.

22. Raposo MT. Incapacidades físicas em hanseníase: avaliação da dimensão do dano através do grau de incapacidade e Eye, Hand, Foot Score. Cad Saúde Colet (Rio J.) 2008; 16:393-4.

23. Orsini MBP. Estudo da função dos nervos periféricos de pacientes com hanseníase, acompanhados por um período médio de 18 anos após o início da poliquimioterapia [Dissertação de Mestrado] Belo Horizonte: Programa de Pós-Graduação em Ciências da Saúde, Universidade Federal de Minas Gerais; 2008.

24. Bandeira RA. Prevalência de hanseníase na macroregião de Palmas, Estado do Tocantins, em 2009 [Dissertação de Mestrado]. Brasília: Universidade de Brasília; 2011.

25. Prata PB, Bohland AK, Vinhas SA. Aspectos epidemiológicos da hanseníase em localidades do Estado de Sergipe, Brasil, período de 1994-1998. Hansenol Int 2000; 25:49-53.

26. Kartikeyan S, Chaturvedi RM. Pattern of leprosy deformities among agricultural labourers in an endemic district: a pilot study. Indian J Lepr 1992; 64:375-9.

27. Opromolla PA, Dalben I, Cardim M. Análise geoestatística de casos de hanseníase no Estado de São Paulo, 1991-2002. Rev Saúde Pública 2006; 40:907-13.
28. Lana FCR, Lanza FM, Velásquez-Meléndez G, Branco AC, Teixeira S, Malaquias LCC. Distribuição da hanseníase segundo sexo no Município de Governador Valadares, Minas Gerais, Brasil. Hansenol Int 2003; 28:131-7.

29. Chichava OA. Fatores associados à baixa adesão ao tratamento da haseníase em pacientes de 78 municípios do Estado do Tocantins [Dissertação de Mestrado]. Fortaleza: Faculdade de Medicina, Universidade Federal do Ceará; 2010.

30. Heukelbach J, Chichava OA, Oliveira AR, Häfner $\mathrm{K}$, Walther F, Alencar CHM, et al. Interruption and defaulting of multidrug therapy against leprosy: population-based study in Brazil's Savannah Region. PLoS Negl Trop Dis 2011; 5:1031.

31. Campos SSL, Ramos Jr. AN, Kerr-Pontes LRS, Heukelbach J. Epidemiologia da hanseníase no Município de Sobral, Estado do Ceará-Brasil, no período de 1997 a 2003 . Hansenol Int 2005; 30:167-73.

32. Secretaria de Estado da Saúde, Governo do Estado de Minas Gerais. Como reconhecer e tratar reações hansênicas. 2a Ed. Belo Horizonte: Governo do Estado de Minas Gerais; 2007.

33. Oliveira CR, Alencar MJF, Santana SC, Sena Neto SA, Ramos Jr. AN. Fatores que influenciaram a ina dequação do diagnóstico e do acompanhamento dos estados reacionais em hanseníase no Estado de Rondônia, Brasil. Hansenol Int 2007; 32:185-96.

34. Grossi MAF, Leboeuf MAA, Reyna MGB, Magalhães ESB, Lana FCF, Bloch KV. Caracterização da demanda pós-alta de hanseníase em serviços de saúde Minas Gerais - Brasil: contribuição para a sistematização da assistência. Cad Saúde Colet (Rio J.) 2009; 17:13-24

35. Duerksen F. Reabilitação. In: Opromolla DVA, Baccarelli R, organizadores. Prevenção de incapacidades e reabilitação em hanseníase. Bauru: Instituto Lauro de Souza Lima; 2003. p. 3-4.

36. Gonçalves SD, Sampaio RF, Antunes CMF. Fatores preditivos de incapacidades em pacientes com hanseníase. Rev Saúde Pública 2009; 43:267-74.

37. Nicholls PG, Croft RP, Richardus JH, Withington SG, Smith WC. Delay in presentation, an indicator for nerve function status at registration and for treatment outcome: the experience of the Bangladesh Acute Nerve Damage Study cohort. Lepr Rev 2003; 74:349-56.

38. Moschioni C, Antunes CM, Grossi MA, Lambertucci JR. Risk factors for physical disability at diagnosis of 19,283 new cases of leprosy. Rev Soc Bras Med Trop 2010; 43:19-22.

39. Oliveira CR, Alencar MJ, Sena Neto SA, Lehman LF, Schreuder PA. Impairments and Hansen's disease control in Rondonia state, Amazon region of Brazil. Lepr Rev 2003; 74:337-48.

40. Alencar CH, Barbosa JC, Ramos Jr. AN, Alencar MJF, Pontes RJS, Castro CGJ, et al. Hanseníase no Município de Fortaleza, CE, Brasil: aspectos epidemiológicos e operacionais em menores de 15 anos (1995-2006). Rev Bras Enferm 2008; 61(esp): 694-700.

Recebido em 19/Jun/2012

Versão final reapresentada em 20/Dez/2012

Aprovado em 08/Jan/2013 